\title{
MOA: A Real-Time Analytics Open Source Framework
}

\author{
Albert Bifet ${ }^{1}$, Geoff Holmes ${ }^{1}$, Bernhard Pfahringer ${ }^{1}$, Jesse Read ${ }^{1}$, \\ Philipp Kranen ${ }^{2}$, Hardy Kremer ${ }^{2}$, Timm Jansen², and Thomas Seidl ${ }^{2}$ \\ 1 Department of Computer Science, University of Waikato, Hamilton, New Zealand \\ \{abifet, geoff, bernhard, jmr30\}@cs. waikato.ac.nz \\ 2 Data Management and Exploration Group, RWTH Aachen University, Germany \\ $\{$ kranen, kremer, jansen, seidl\}@cs.rwth-aachen.de
}

\begin{abstract}
Massive Online Analysis (MOA) is a software environment for implementing algorithms and running experiments for online learning from evolving data streams. MOA is designed to deal with the challenging problems of scaling up the implementation of state of the art algorithms to real world dataset sizes and of making algorithms comparable in benchmark streaming settings. It contains a collection of offline and online algorithms for classification, clustering and graph mining as well as tools for evaluation. For researchers the framework yields insights into advantages and disadvantages of different approaches and allows for the creation of benchmark streaming data sets through stored, shared and repeatable settings for the data feeds. Practitioners can use the framework to easily compare algorithms and apply them to real world data sets and settings. MOA supports bi-directional interaction with WEKA, the Waikato Environment for Knowledge Analysis. Besides providing algorithms and measures for evaluation and comparison, MOA is easily extensible with new contributions and allows for the creation of benchmark scenarios.
\end{abstract}

\section{Introduction}

In data stream scenarios data arrives at high speed strictly constraining processing algorithms in space and time. To adhere to these constraints, specific requirements have to be fulfilled by the stream processing algorithms, that are different from traditional batch processing settings. The most significant requirements are the following: process an example at a time, and inspect it at most once; use a limited amount of memory; work in a limited amount of time; and be ready to predict at any time.

Stream learning algorithms are an important type of stream processing algorithm: in a repeated cycle, the learned model is constantly updated to reflect the incoming examples from the stream. They do so without exceeding their memory and time bounds. After processing an incoming example, the algorithms are always able to output a model. Typical learning tasks in stream scenarios are classification, regression, clustering, and frequent pattern mining. 
MOA is an open-source framework for dealing with massive evolving data streams. It is the first data mining software designed specifically for data streams to include multi-label classification and graph mining methods, in addition to regular classification and clustering methods [3].

Our stream learning framework provides a set of data generators, algorithms and evaluation measures. Practitioners can benefit from this by comparing several algorithms in real world scenarios and choosing the best solution. For researchers our framework yields insights into advantages and disadvantages of different approaches and allows the the creation of benchmark streaming data sets through stored, shared and repeatable settings for the data feeds. The sources are publicly available and are released under the GNU GPL license.

Only two other open-source data streaming packages exist: VFML and a RapidMiner plugin. The VFML (Very Fast Machine Learning) [4 toolkit was the first open-source framework for mining high-speed data streams and very large data sets. It was developed until 2003. VFML is written mainly in standard C, and contains tools for learning decision trees (VFDT and CVFDT), for learning Bayesian networks, and for clustering.

The data stream plugin (formerly: concept drift plugin) [5] for RapidMiner (formerly: YALE (Yet Another Learning Environment)), is an extension to RapidMiner implementing operators for handling real and simulated concept drift in evolving streams.

MOA is built on experience with both WEKA and VFML. The main advantage of MOA is that it provides many of the recently developed data stream algorithms, including learners for multi-label classification and graph mining. It also contains a graphical interface, and the software is built using object-oriented techniques. Generally, it is straightforward to use or to extend MOA.

\section{Experimental Framework}

MOA is written in Java. The main benefits of Java are portability, where applications can be run on any platform with an appropriate Java virtual machine, and the strong and well-developed support libraries. Use of the language is widespread, and features such as automatic garbage collection help to reduce programmer burden and error.

MOA contains stream generators, learners and evaluation methods. Figure 1 shows the MOA graphical user interface. However, a command line interface is also available. Considering data streams as data generated from pure distributions, MOA models a concept drift event as a weighted combination, or mixture distribution, of two pure distributions that characterize the target concept before and after the drift. The mixing proportion for the "after" concept smoothly increases from zero to one inside a user-defined window around the time point of change. The increase follows a sigmoid function, an elegant and practical solution [2].

MOA streams can be built using generators, reading ARFF files, joining several streams, or filtering streams. Most of the data generators commonly found 

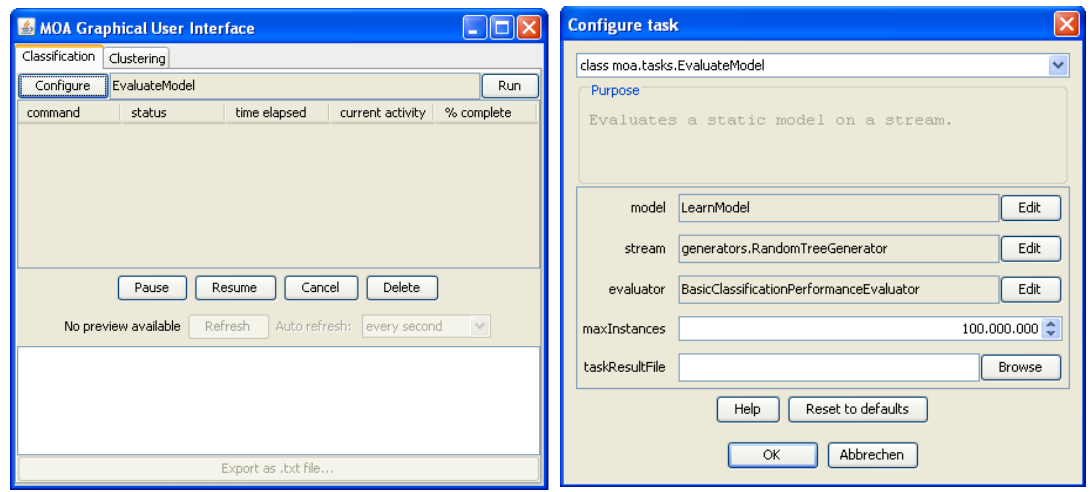

Fig. 1. MOA Graphical User Interface

in the literature, are provided: Random Tree Generator, SEA Concepts Generator, STAGGER Concepts Generator, Rotating Hyperplane, Random RBF Generator, LED Generator, Waveform Generator, and Function Generator.

MOA contains a range of classification methods such as: Naive Bayes, Stochastic Gradient Descent, Perceptron, Hoeffding Tree, Adaptive Hoeffding Tree, Hoeffding Option Tree, Bagging, Boosting, Bagging, and Leveraging Bagging.

For clustering 6], MOA contains several stream clustering methods such as StreamKM++, CluStream, ClusTree, Den-Stream, and CobWeb. Dynamic visualization of cluster evolution is available, as depicted in Figure 2.

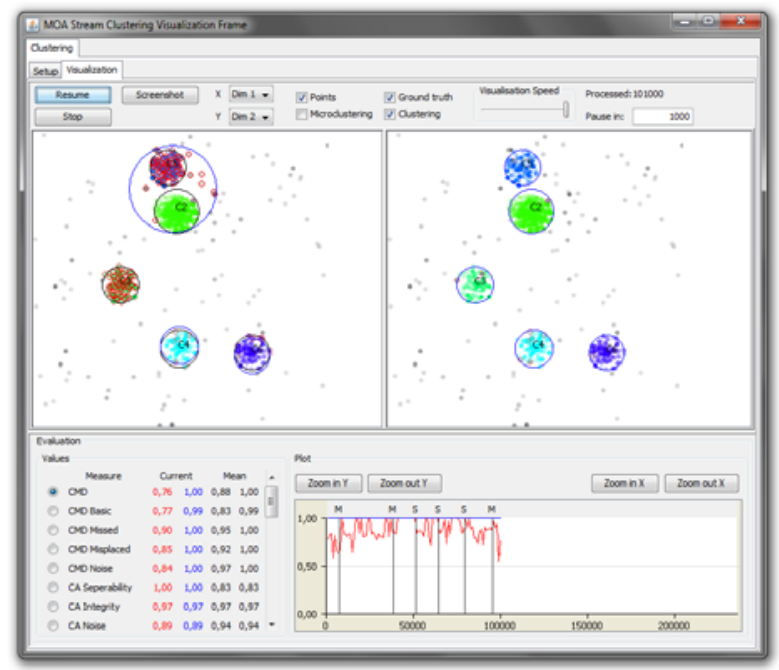

Fig. 2. Visualization tab of the clustering MOA graphical user interface 
Two recent extensions to MOA are multi-label classification and graph mining. In multi-label classification, instead of a single class-label, each example can be associated with multiple labels. Multi-label classification has seen considerable development in recent years, but so far most of this work has been carried out in the context of batch learning where train-then-test or cross-fold validation evaluations are typical. MOA implements multi-label stream generators and several state of the art methods: ECC Ensembles of classifier-chains, and EPS Ensembles of Pruning Sets, Multi-label Hoeffding Trees, and multi-label adaptive bagging methods.

MOA also contains a framework for studying graph pattern mining on time-varying streams [1. All methods work on coresets of closed subgraphs, compressed representations of graph sets, and maintain these sets in a batchincremental manner, but use different approaches to address potential concept drift. MOA implements InCGRAPHMiner, WinGraphMiner and ADAGraphMineR.

\subsection{Website, Tutorials, and Documentation}

MOA can be found at: http://moa.cs.waikato.ac.nz/ The website includes a tutorial, an API reference, a user manual, and a manual about mining data streams. Several examples of how the software can be used are available. We are currently working on extending the framework to include data stream regression, and frequent pattern learning.

Acknowledgments. This work has been supported by the UMIC Research Centre, RWTH Aachen University, Germany.

\section{References}

1. Bifet, A., Holmes, G., Pfahringer, B., Gavaldà, R.: Mining frequent closed graphs on evolving data streams. In: 17th ACM SIGKDD (2011)

2. Bifet, A., Holmes, G., Pfahringer, B., Kirkby, R., Gavaldà, R.: New ensemble methods for evolving data streams. In: 15th ACM SIGKDD (2009)

3. Bifet, A., Holmes, G., Pfahringer, B., Kranen, P., Kremer, H., Jansen, T., Seidl, T.: Moa: Massive online analysis, a framework for stream classification and clustering. Journal of Machine Learning Research - Proceedings Track 11, 44-50 (2010)

4. Hulten, G., Domingos, P.: VFML - a toolkit for mining high-speed time-changing data streams (2003)

5. Klinkenberg, R.: Rapidminer data stream plugin. RapidMiner (2010), http://www-ai.cs. uni-dortmund.de/auto?self=\$eit184kc

6. Kremer, H., Kranen, P., Jansen, T., Seidl, T., Bifet, A., Holmes, G., Pfahringer, B.: An effective evaluation measure for clustering on evolving data stream. In: 17 th ACM SIGKDD (2011) 